\title{
Oddity learning in the pigeon: Effect of negative instances, correction, and number of incorrect alternatives
}

\author{
THOMAS R. ZENTALL \\ University of Kentucky, Lexington, Kentucky 40506 \\ DAVID E. HOGAN \\ Northern Kentucky University, Highland Heights, Kentucky 41076 \\ and \\ CHARLES A. EDWARDS \\ University of Kentucky, Lexington, Kentucky 40506
}

\begin{abstract}
Pigeons have difficulty learning a standard oddity task involving two colors and three stimulus positions. In Experiment 1, performance on standard noncorrection trials was compared with performance on (1) rerun correction trials in which errors resulted in trial repetition, (2) noncorrection trials with added "negative instance" trials involving presentation of three stimuli, all of which matched, and (3) a combination of correction and added negative instance trials. Results indicated that negative instances, but not correction trials, significantly facilitated oddity performance. In Experiment 2, Phase 1, number of stimulus positions lit (three or five) was factorially manipulated with number of positions on which the odd stimulus could appear (three or five). An increase in number of positions lit, but not number of positions that could be odd, facilitated performance. In Phase 2, birds transferred from trials with five positions lit to four positions lit performed significantly better than controls; but in Phase 3 , the same birds did not perform significantly better than controls when transferred to trials with three positions lit. In both experiments, analysis of performance as a function of response position indicated better performance at the end of each display than in the middle. These results, together with the group performance differences in Experiment 2, suggest that oddity learning in pigeons involves a size, or number, discrimination.
\end{abstract}

The standard oddity task is widely used in the study of comparative animal learning. It is a task that has been used to assess animal intelligence because some species appear to learn it readily, others learn it slowly, and still others may not be able to learn it at all (see Zentall, Hogan, \& Holder, 1974, for a brief review). Standard oddity generally involves the simultaneous presentation of three stimuli. Reinforcement is provided for responding to the one that is different from the other two. Typically, the stimuli take on one of two values (e.g., red or green), which are counterbalanced such that stimulus or position preferences cannot result in a high level of performance.

Zentall et al. (1974) have found that pigeons' performance of the standard oddity task, involving red and green stimuli presented on a typical three-key intelligence panel, did not rise above $50 \%$ correct.

This research was supported by Grant MH24092 from the National Institute of Mental Health to the first author. Requests for reprints should be sent to Thomas $R$. Zentall, Department of Psychology, University of Kentucky, Lexington, Kentucky 40506.
The task was learned, however, when the odd stimulus could appear only on either end position. This modification of the standard oddity task is actually a variation of oddity-from-sample in which responses to a singly lit center key illuminate the side keys, and reinforcement is provided for a peck to that stimulus which is different from the center stimulus. According to Zentall et al., when the center key is never odd it can provide information about the incorrect color on each trial. If, in fact, this information is used, it suggests that the oddity-from-sample task (with or without required pecks to the sample) and the standard oddity task are learned in different ways. The fact that Zentall et al. found poor transfer from oddity-from-sample to standard oddity supports the hypothesis that the two tasks are quite different.

Zentall et al. also found that pigeons exposed to standard oddity training initially performed below $50 \%$ correct due to strong position preferences (with three response keys, chance was $33 \%$ correct). Over the course of the first few sessions of training, however, performance rose to $50 \%$ correct, due to a shift from a position preference to a stimulus (color) preference 
(two colors were used and each was odd on half the trials), and remained at that level for 65 training sessions. Zentall et al. reasoned that, although it was not clear whether stimulus preferences were responsible for failure to learn oddity or were merely a symptom of the failure to find a "better solution," pigeons' oddity performance might benefit from the elimination of stimulus preferences.

One means of eliminating stimulus preferences is to require a "corrected" response before the next trial begins. Zentall et al. exposed birds to a rerun correction procedure in which an incorrect response resulted in rerunning the trial. The results indicated that stimulus preferences were greatly reduced, and performance levels increased significantly. But since the procedure was initiated only after 65 sessions of noncorrection training, no control group was included, and performance appeared to level off at about $60 \%$ correct, it is difficult to evaluate the magnitude and reliability of correction training on standard oddity learning. Furthermore, the bulk of the animal literature suggests that correction training retards acquisition. One purpose of the present study was to compare the effect of oddity correction training administered from the start of training with the effect of oddity noncorrection training.

A second variable studied in the present investigation was the presence or absence of "negative instances" of oddity trials. Zentall and Hogan (1978) found that transfer of both matching-to-sample and oddity-from-sample to new stimuli was improved when, during original training, trials were included on which neither alternative was correct (i.e., for matching birds, neither comparison stimulus matched the sample; for oddity birds, both comparison stimuli matched the sample and the trial would not terminate until $3 \mathrm{sec}$ had passed without a response to either comparison stimulus). Zentall and Hogan did not assess the effect of negative instances on acquisition of matching-to-sample and oddity-from-sample; rather, they introduced negative instance trials only after both tasks had been learned to high asymptotic levels. Zentall and Hogan suggested that training with negative instances might allow for better concept learning to occur, though it is also possible that negative instances forced the pigeons to examine all of the stimulus alternatives. In either case, such trials might promote learning of standard oddity.

In order to further facilitate oddity learning, a change was made in the apparatus. In the standard three-key operant chamber used by Zentall et al., the keys were $6.0 \mathrm{~cm}$ apart (edge to edge), a distance that may not have encouraged the pigeons to make comparisons among the keys. In the present study, square, contiguous pecking keys were used.

If standard oddity learning is a viable means of comparing intelligence among species, then a thorough analysis of the task and an understanding of how the task is learned may suggest that different species apply different strategies or engage in different processing of the stimulus display in attempting to master the task. Thus, the present experiment sought to determine conditions under which standard oddity could be learned, and possible mechanisms underlying such learning.

\section{EXPERIMENT 1}

\section{Method}

\section{Subjects}

Sixteen male White Carneaux pigeons were deprived to $75 \%-80 \%$ of their free-feeding weights. Twelve of the birds had had prior shape discrimination training but no prior experience with colors. The remaining four were experimentally naive. Lights were on in the colony room from $0600-1800 \mathrm{~h}$ daily.

\begin{abstract}
Apparatus
Experimental sessions were conducted in a standard pigeon operant chamber that was $35 \mathrm{~cm}$ high and $30 \mathrm{~cm}$ deep on the inside and $35 \mathrm{~cm}$ across the intelligence panel. The bottom edge of a centrally mounted grain feeder was $9.5 \mathrm{~cm}$ from the floor. Whenever the grain feeder was raised it was illuminated by a 28-V .04-A lamp. Above the feeder were five square pecking keys $(2.5 \times 2.5 \mathrm{~cm})$ mounted side by side behind a $12.5 \times 2.5 \mathrm{~cm}$ slot in the panel. The keys were separated by sheet metal shims that projected $.6 \mathrm{~cm}$ beyond the surface of the keys. The bottom edge of the keys was $16.0 \mathrm{~cm}$ from the floor. Behind each pecking key was an in-line projector with 28-V .1-A lamps that could illuminate the key with a red or green field (Kodak Wratten filters 26 and 60, respectively). Control equipment was located in an adjoining room. A dim houselight was located above the pecking keys. Masking of sounds was provided by white noise and a blower fan.
\end{abstract}

\section{Procedure}

Pretraining. All birds were magazine trained (or, in the case of experienced birds, magazine tested) and then shaped to peck one of the middle three keys that was either red or green. The position and color of the shaping key were roughly counterbalanced over birds. On the following day, each bird was given 48 reinforcements (2 sec access to mixed Purina pigeons grains), one for each response to a lit key, which changed position (any one of the three middle positions) and color (red or green) randomly following each reinforcement. On the next day, another 48 pretraining trials were given, but five pecks were required to each stimulus before reinforcement was provided.

Training. On the following day, the birds were assigned randomly to four groups, with the constraint that each of the four experimentally naive birds be assigned to a different group. All birds were exposed to standard oddity trials involving red and green stimuli presented on the three middle response keys. Trials were of two general types: two reds and a green, or two greens and a red. The odd stimulus appeared on any one of the three middle positions. Five pecks terminated a trial. If the fifth peck was made to the odd stimulus, the trial ended with reinforcement. Pecks to the darkened end keys had no programmed consequence. The houselight was lit during all trials. Trials were separated by a darkened 5 -sec intertrial interval.

Group NC (noncorrection) was exposed to standard oddity trials. The position and the color of the odd stimulus varied from trial to trial regardless of whether a correct response had been made. The order was random except that (1) color and position of the odd stimulus were counterbalanced over trials, and (2) on no more 
than three consecutive trials was the same color or same position correct.

Group C (correction) was exposed to standard oddity trials, but a change in trial type occurred only following a correct response. Following an incorrect response, the trial was repeated.

Group NCNI (noncorrection, negative instance) was exposed to noncorrection standard oddity trials among which were interspersed 48 negative instance trials. On negative instance trials, the three response keys were lit either all red or all green and the keys remained lit until $3 \mathrm{sec}$ had passed without a response to any of the keys. Reinforcement was not provided on negative instance trials. The intertrial interval began with the offset of the keys.

Group CNI (correction, negative instance) was exposed to correction standard oddity trials among which were interspersed 48 negative instance trials. Because there was an inherent correction procedure on negative instance trials (i.e., negative instance trials would not terminate until $3 \mathrm{sec}$ had passed without a response), the rerun correction procedure applied only to oddity trials.

For all four groups, each session was defined by 48 reinforcements. Experiment 1 consisted of 100 training sessions.

\section{Results}

The rates of oddity learning, ordered from fastest to slowest, were Group NCNI, Group CNI, Group C, and Group NC. The learning curves appear in Figure 1 . For the correction groups, the data represent the percentage of trials correct on the first attempt. For the noncorrection groups, the data represent the percentage of trials correct.

A two-way analysis of variance performed on the data pooled over the training sessions indicated that the difference in performance between the groups that were exposed to negative instance trials and those that were not was significant $[F(1,12)=7.46$; the .05 level of significance applied to all analyses]. The difference in performance between correction groups and noncorrection groups, however, was not significant $(F<1)$. Although the data in Figure 1 suggest that there may have been an interaction between the two training variables, the analysis indicated that the

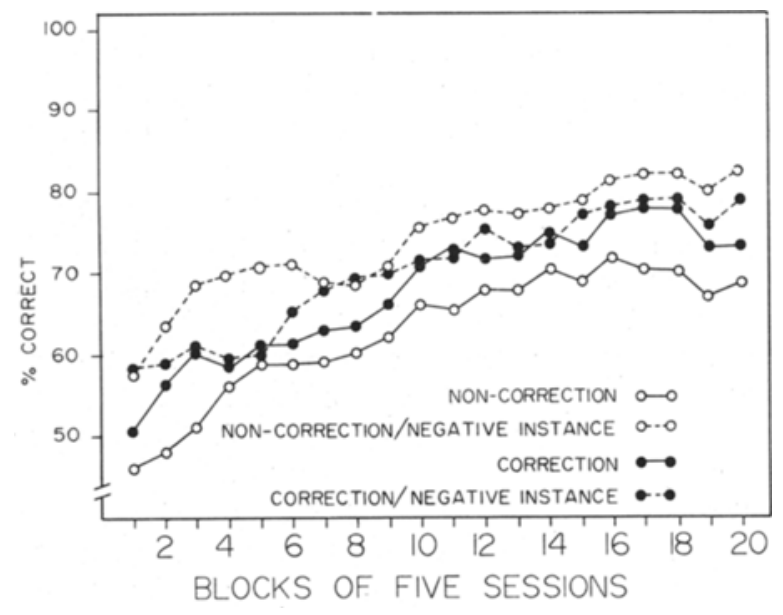

Figure 1. Experiment 1: Oddity acquisition by Groups NC (noncorrection), $\mathrm{C}$ (correction), NCNI (noncorrection with negative instance trials), and CNI (correction with negative instance trials). effect was not reliable $[F(1,12)=2.96]$. Thus, the training data indicate that only negative instance training had a significant effect on oddity learning.

The above analyses were performed on data equated for number of reinforcements per session. The procedure used allowed for number of trials per session to vary. To the extent that correction birds engaged in perseverative responding to position or color, correction birds could have received more training trials than the noncorrection birds. Examination of trialsper-session scores suggested, however, that perseverative responses by correction birds dropped out within the first few sessions. A two-way analysis of variance performed on the total number of oddity training trials for each bird failed to disclose a significant difference between correction and noncorrection groups $[F(1,12)=1.67]$. A significant difference in total number of oddity trials was found, however, between birds that had received negative instance trials and those that had not $[F(1,12)=5.89]$. The negative instance groups had significantly fewer oddity training trials than the groups not exposed to negative instance trials. The difference in the number of total oddity training trials was directly related to performance differences mentioned earlier, because reinforcements per session were held constant. The interaction between negative instance training and correction training was also reliable $[F(1,12)=9.95]$. Apparently, negative instance training results in the need for fewer oddity training trials only when a noncorrection procedure is used.

Although negative instance training clearly facilitates oddity performance, one can ask if such training would be an efficient procedure for acquiring oddity; that is, would the birds have benefited as much from a comparable number of oddity trials. The present design makes it difficult to answer the question of efficiency directly because total trials per session was free to vary and was inversely related to performance. It may not be appropriate to compare group performance at different points in time and with practice distributed differently. One can get an approximate measure of efficiency, however, by (1) calculating the mean total number of trials experienced by birds not exposed to negative instance trials, (2) finding the session on which each negative instance bird reached that number of oddity and negative instance trials combined, and (3) comparing performance by the two groups over a comparable number of total trials. A two-way analysis of variance indicated that when total trials were equated, performance differences attributable to negative instance experience were marginally significant $[F(1,12)=3.87, p=.07]$. Thus, negative instance trials not only facilitate oddity acquisition but also appear to be more effective than a comparable number of oddity trials.

Zentall et al. (1974) observed that, even though standard oddity performance was poor, performance 
Table 1

Mean Number of Trials Correct at Each Response Position (Experiment 1)

\begin{tabular}{lccc}
\hline Group & Position 2 & Position 3 & Position 4 \\
\hline NC & 21.4 & 6.4 & 20.2 \\
C & 13.7 & 8.5 & 13.2 \\
NCNI & 19.6 & 9.2 & 19.2 \\
CNI & 15.7 & 8.2 & 13.8 \\
\hline
\end{tabular}

Vote-Sum of mean correct across response positions for each noncorrection bird is always 48 , but for each correction bird it is a maximum of 48 because corrected responses were not counted as correct.

on the center key was inferior to performance on the two end keys. As can be seen in Table 1, which is based on the last 10 training sessions, a similar effect was found in the present study for all groups. Mean number of trials correct at each position have been presented, rather than percentage correct, because birds were equated for total reinforcements received rather than total trials presented. To assess the reliability of performance differences on the three keys, a correlated-difference $t$ test was performed on the data from each group. The difference between end-key performance and center-key performance was significant for each of the four groups [t(3)= $4.23,5.55,5.46$, and 7.24 , for NC, C, NCNI, and CNI, respectively]. A second test indicated that the difference between the two end-key scores was not reliable for any of the four groups $[\mathrm{t}(3)=.77, .48,3.16$, and 2.52, for NC, C, NCNI, and CNI, respectively].

Analyses of variance were then performed on the difference scores to determine the effect of treatment on the magnitude of the position effect. Analysis of the first difference scores (end vs. center key performance) indicated a significant effect of correction training $[F(1,12)=10.39]$, but a nonsignificant effect of negative instance training $(F<1)$ and a nonsignificant interaction $[F(1,12)=2.15]$. The correction training effect indicated that correction training produced shallower $\mathrm{V}$-shaped position functions and was thus successful in reducing strong stimulus preferences.

Analysis of the second difference scores (left- vs. right-key performance) indicated no significant effect of either correction or negative instance training (both $\mathrm{Fs}<1$ ) and no significant interaction $[\mathrm{F}(1,12)$ $=1.14]$.

\section{Discussion}

The results of Experiment 1 indicate that negative instance trials can have a significant facilitative effect on standard oddity learning and that inclusion of these trials may even produce a more efficient procedure for training standard oddity.
In an earlier experiment, Zentall and Hogan (1978) found that negative instance training facilitated the development of generalized matching-to-sample and oddity-from-sample concepts. In Zentall and Hogan's experiments, it was assumed that the negative instances better defined the concepts by forcing the birds to actively avoid responding to incorrect alternatives. Thus, the negative instances perhaps better identified the concepts boundary conditions. It is possible that negative instance training played the same role in the present experiment. If a generalized oddity concept was developed by birds trained with negative instances, one would expect concept generalization from performance with one stimulus odd to performance with the other stimulus odd. Such generalized concept learning should result in faster acquisition of the standard oddity task.

It is also possible that negative instance training encouraged the birds to observe all three alternatives by penalizing them for pecking on negative instance trials (the negative display did not terminate). More simply stated, negative instance training may have caused the birds to delay responding, an outcome that reduced the likelihood of "impulsive" errors (i.e., errors resulting from the incomplete observation of all three keys).

A third way in which negative instance training could have facilitated oddity learning is by providing perceptually contrasting trials. The odd stimulus may stand out better if it is presented in a context of being absent on some trials. Thus, the unique aspect of the oddity trials (i.e., having an odd stimulus) may have been made more salient.

Whatever the underlying mechanism, it is clear that negative instance training can have beneficial effects on acquisition and transfer of certain conditional discrimination tasks. An understanding of the mechanism responsible for such facilitation could lead to a better understanding of how conditional discriminations, in general, are learned.

Although negative instance training led to faster oddity acquisition, correction training did not significantly facilitate standard oddity learning in the present study. Zentall et al. (1974), however, found significant facilitation of oddity performance when birds were exposed to correction trials. The difference in outcome may be related to the fact that control birds in the present study (Group NC) showed substantial oddity learning, whereas a comparable group in Zentall et al.'s experiment showed little sign of learning. The latter study reported facilitation due to correction training as an improvement in performance from a 65 -session baseline of about $50 \%$ correct responding to over $60 \%$ correct responding within 10 sessions, but no further improvement over the next 5 sessions. Perhaps correction training allows 
oddity learning to emerge if it has been obscured by strong position and color preferences, which Zentall et al. reported were present, but has little direct effect on oddity learning.

The reason for the difference in performance between control groups in the two studies was probably related to the fact that Zentall et al. used a standard chamber with three separated pecking keys, whereas the present study used three contiguous keys, a change intended to maximize the opportunity for comparison among the keys.

Although the present experiment did not replicate the effect of correction training on oddity performance found by Zentall et al., the present experiment (Groups $\mathrm{C}$ and $\mathrm{NC}$ ) indicated a difference in performance in the same direction [albeit not significant, $F(1,6)=1.88$ ] as that found earlier. Thus, the results of both experiments are inconsistent with the body of data that indicate that correction training leads to slower acquisition than noncorrection training (see Zentall et al. for a review). As Zentall et al. proposed, correction training may affect complex tasks (e.g., conditional discriminations) differently from simple discrimination tasks.

In addition to between-groups differences, large within-group differences in performance as a function of key position were found during the course of the experiment. The performance differences should provide some indication of how pigeons experience the standard oddity task and what mechanisms are involved in oddity learning.

One means of mastering the oddity task is to view it as a size or number discrimination; a response to the stimulus color of the smaller area, or stimulus color that is least often represented on a trial, is reinforced. If oddity learning reduces to a size-number discrimination, one would expect the center-odd trials to be more difficult than the end-odd trials. With the odd stimulus on the center, the larger area is divided into two smaller areas that might be less easily integrated or counted; that is, center-odd trials would be expected to break up the organization or grouping of incorrect alternatives. This prediction follows directly from the gestalt principle of grouping by spatial proximity (see Koffka, 1935, pp. 164167).

Alternatively, the oddity task can be viewed as a figure-ground problem (see Koffka, 1935, chap. 5) with the odd stimulus serving as the figure and the two matching stimuli as the ground. If oddity learning involved a perceptual figure-ground relation, then the center-odd trials should be somewhat easier than the end-odd trials. On center-odd trials, the matching stimuli surround the odd stimulus, and thus should make it stand out better.

The position data suggest that, under the present conditions, the birds learned a size or number dis- crimination. Performance on center-odd trials was consistently lower than performance on end-odd trials. In fact, performance level on center-odd trials averaged (over the four groups) only .49 of performance level on end-odd trials.

\section{EXPERIMENT 2}

Further relevant data could be obtained by directly manipulating size-number, that is, by increasing the number of incorrect alternatives. According to the sizenumber-discrimination hypothesis, an increase in the number of incorrect alternatives from two to four should result in facilitated learning. With four incorrect alternatives, there would always be at least two contiguous incorrect alternatives on each trial. With only two incorrect alternatives, however, both incorrect alternatives would be contiguous on only twothirds of the trials.

Although the consequences of such a manipulation are not so readily predicted from the figure-ground hypothesis, facilitated learning with an increase in the number of incorrect alternatives can be explained. With more incorrect alternatives, there should he a greater tendency for the odd stimulus to stand out against the larger ground. Again, the position data should distinguish between the two theories. The sizenumber hypothesis would predict better performance at the ends of the display, whereas the figure-ground hypothesis would predict better performance at the center of the display.

There is evidence from a variety of species that an increase in the number of incorrect alternatives has a facilitative effect on oddity learning. The effect has been found in children (Gollin, Saravo, \& Salten, 1967; Gollin \& Schadler, 1972), chimpanzees (Nissen \& McCulloch, 1937), and canaries (Pastore, 1954). Unfortunately, studies that have manipulated number of incorrect alternatives have not provided the position data with which to evaluate the two odditylearning hypotheses.

The purpose of Experiment 2, Phase 1 was to assess the generality of earlier findings of facilitated performance with an increase in the number of incorrect alternatives and, if facilitation occurred, to examine the position data to determine its source.

The relationship between two-incorrect-alternative oddity and many-incorrect-alternative oddity was further examined in Experiment 2, Phases 2 and 3 by assessing transfer from the easier task to the harder task. If the two tasks are learned in the same way, one would expect a high level of performance by birds transferred from many-incorrect-alternative to two-incorrect-alternative oddity. If, however, the two-incorrect-alternative oddity task is uniquely difficult because it includes center-odd trials on which birds must combine the information present on the 
two end keys in order to perform above chance, one might expect a drop in performance when the birds are transferred.

In Phases 2 and 3 of Experiment 2, the number of incorrect alternatives was gradually reduced so as to minimize the effect on performance of generalization decrement.

\section{Method}

\section{Suhjects and Apparatus}

Subjects and apparatus were the same as those in Experiment 1.

\section{Procedure}

Phase 1. Each of the 16 birds from Experiment 1 was assigned to one of four groups, counterbalanced for condition experienced and equated for terminal performance. Group $\mathrm{Cl}$ was exposed to standard oddity trials identical to those experienced by Group NC in Experiment 1. Group C2 was also exposed to standard oddity trials involving three lit adjacent keys on each trial; but the particular three keys that were lit changed from trial to trial, such that on a given trial the odd stimulus could appear on any one of the five response keys. Group E1 was exposed to trials on which all five response keys were lit, but on a given trial the odd stimulus could appear only on the three middle response keys. Thus, the end keys were lit on each trial but the odd stimulus never appeared there. Group E2 was exposed to trials on which all five response keys were lit, and on a given trial the odd stimulus could appear on any one of the five keys.

Comparison of data from the two experimental groups (E1 and $\mathrm{E} 2$ ) with the two control groups (C1 and $\mathrm{C2}$ ) allows one to assess the effect on oddity learning of increasing the number of incorrect alternatives. Comparison of data from Group E1 with the other groups allows one to assess the effect on oddity learning of providing, at a predictable location, a cue indicating which color is incorrect on each trial. Group $\mathrm{C} 2$ served as a control for simply increasing the number of response keys to which pecks could be reinforced, a manipulation intended to reduce absolute position responding and potentially facilitate oddity performance. Phase 2 consisted of 18120 -noncorrection-trial sessions.

Phase 2. Group E1 was transferred to a four-key-lit-three-keyodd task. The end key on the left was never lit, and the end key on the right was never odd. Group E2 was transferred to a four-key-lit-three-key-odd task. The end key on the left was never lit, and the odd stimulus was presented on any of the remaining four keys. Group C2 was transferred to the three-key-litthree-key-odd task. Only the three middle keys were lit, and any one of the three could be odd. Group $\mathrm{Cl}$ continued with the threekey-lit-three-key-odd task. Phase 3 consisted of 12120 -trial sessions.

Phase 3. All pigeons were transferred to the three-key-lit-threekey-odd task for 10120 -trial sessions.

The complete design of Experiment 2 appears in Table 2.

\section{Results}

\section{Phase 1}

The Phase 1 data indicate that Group E1 performed somewhat better than Group E2 and that Group E2 performed better than the two control groups. Initial differences in performance between the two control groups were found, but they dissipated with practice. The data from Phase 1 appear in Figure 2.

A two-way analysis of variance performed on the data from the first session of Phase 1 indicated that performance was better with five keys lit (Groups E1
Table 2

Design of Experiment 2

\begin{tabular}{|c|c|c|c|c|c|c|}
\hline \multirow[b]{2}{*}{ Group } & \multicolumn{2}{|c|}{ Phase 1} & \multicolumn{2}{|c|}{ Phase 2} & \multicolumn{2}{|c|}{ Phase 3} \\
\hline & $\mathrm{L}$ & 0 & $\mathrm{~L}$ & 0 & $\mathbf{L}$ & 0 \\
\hline $\mathrm{C} 1$ & 3 & 3 & 3 & 3 & 3 & 3 \\
\hline $\mathrm{C} 2$ & 3 & 5 & 3 & 3 & 3 & 3 \\
\hline E1 & 5 & 3 & 4 & 3 & 3 & 3 \\
\hline E2 & 5 & 5 & 4 & 4 & 3 & 3 \\
\hline
\end{tabular}

Note $-L=$ number of keys lit on each trial; $O=$ number of keys that could be odd across trials.

and E2) than with three keys lit (Groups $\mathrm{Cl}$ and $\mathrm{C2}$ ) $[F(1,12)=12.88]$. Increasing the number of keys that could be odd from three (Groups El and C1) to five (Groups E2 and C2) appeared to disrupt performance, although the difference was not quite significant $[F(1,12)=4.48, p=.06]$. The interaction between the number of keys lit and number of keys that could be odd was not reliable $(\mathrm{F}<1)$.

A similar analysis performed on the data pooled over the 18 Phase 1 sessions indicated a significant effect of number of keys lit $[F(1,12)=7.04]$, but the effect of number of keys that could be odd was greatly reduced $[F(1,12)=1.10]$, and again the interaction between number of keys lit and number of keys that could be odd was not reliable.

Because the five-key-lit-three-key-odd task was unique in providing positions that consistently indicated which color was incorrect, a separate analysis compared performance of Group E1 with that of Group E2. The analysis, consisting of data pooled over the 18 Phase 1 sessions, indicated that the two groups differed significantly $[F(1,6)=6.14]$. Since, for Group E1, the odd stimulus appeared only on the middle three keys, it might be more appropriate to compare Group E1's performance with Group E2's performance on the three middle keys. When only performance on the three middle keys was considered, the difference in performance between Groups E1 and E2, pooled over the 18 Phase 1 sessions, was even larger [see Table $3 ; \mathrm{F}(1,6)=8.85$ ]

The position data from Phase 1 pooled over the last 10 sessions indicated that performance was considerably higher at the ends of the display than at the center for Group C1. A similar outcome, though much shallower, was found for Groups E1 and E2. The position data from the last 10 sessions of Phase 1 appear in Table 3. The Phase 1 data from Group C2 do not appear in Table 3 because relative position data were not taken separately and the absolute and relative positions of the odd stimulus were neither isomorphic nor orthogonal; for example, when the center key was odd, it could have been either at the center or at the end of the lit display, but when one of the end keys was odd, it could only have been at the end of the lit display. 


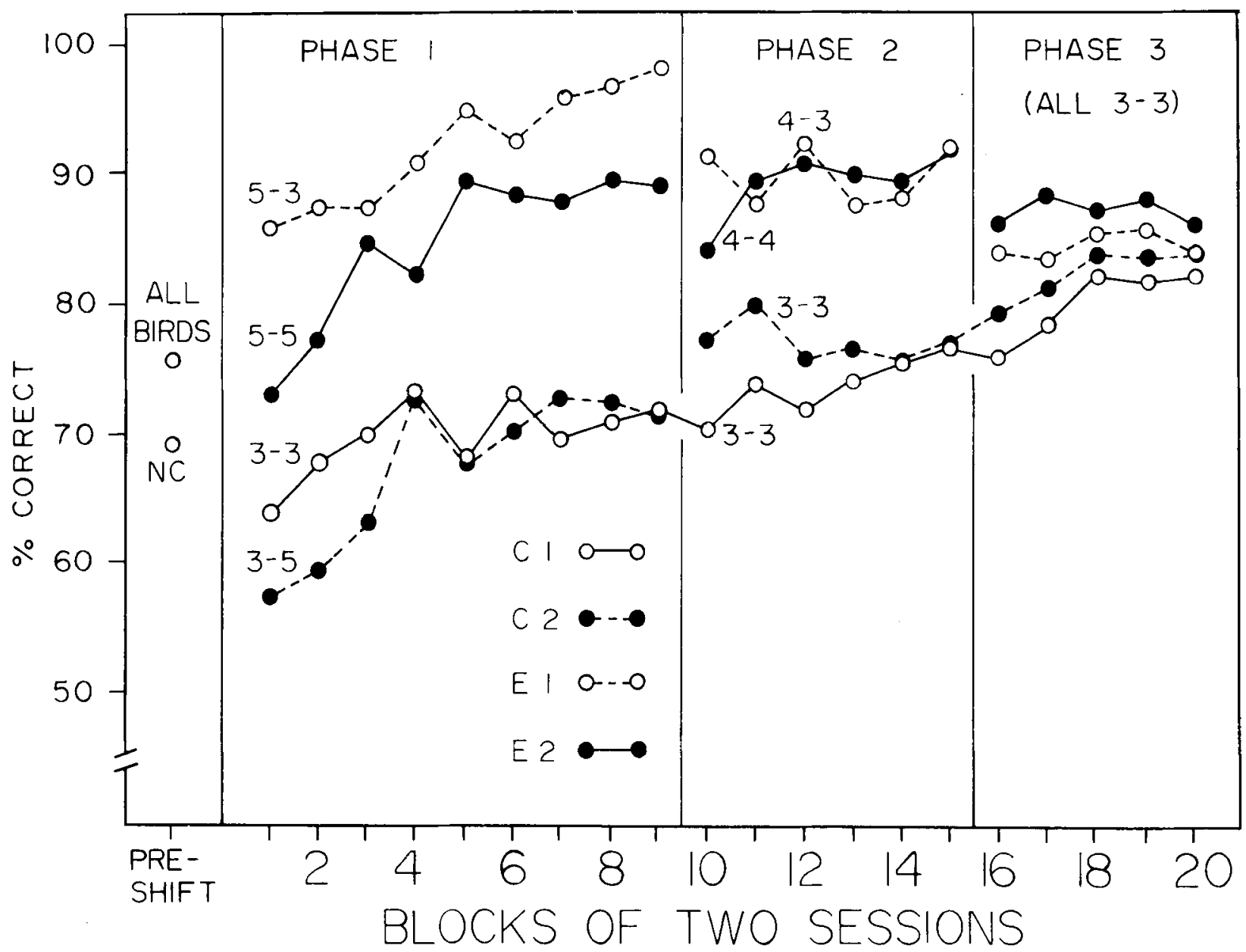

Figure 2. Experiment 2, Phase 1: Oddity performance by Groups $C 1$ (three keys lit and any one of the three keys could be odd), C2 (three keys lit, but the particular three keys varied from trial to trial such that any one of five keys could be odd), E1 (five keys lit, but only the three middle keys could be odd), and E2 (five keys lit and any one of the five keys could be odd). Phase 2: Birds in Group $\mathrm{C} 1$ remained on the three-key-lit-three-key-odd task. Birds in Group $\mathrm{C} 2$ were transferred to the three-key-lit-three-key-odd task. Birds in Group E1 were transferred to a four-key-lit-three-key-odd task. Birds in Group E2 were transferred to a four-key-lit-four-key-odd task. Phase 3: All birds were exposed to the three-key-lit-three-key-odd task. Preshift performances (pooled over the last two sessions of Experiment 1) averaged over all birds and averaged over just the noncorrection condition (NC) are presented in the left panel. The noncorrection procedure of Experiment 1 is directly comparable to the three-key-lit-three-key-odd procedure of Experiment 2.

Table 3

Percentage Correct at Each Response Position (Experiment 2)

\begin{tabular}{|c|c|c|c|c|c|c|c|c|c|c|c|c|c|c|c|c|c|c|}
\hline \multirow[b]{3}{*}{ Group } & \multicolumn{7}{|c|}{ Phase 1} & \multicolumn{6}{|c|}{ Phase 2} & \multicolumn{5}{|c|}{ Phase 3} \\
\hline & \multirow[b]{2}{*}{$\mathbf{L}$} & \multirow[b]{2}{*}{0} & \multicolumn{5}{|c|}{ Position } & \multirow[b]{2}{*}{$\mathbf{L}$} & \multirow[b]{2}{*}{0} & \multicolumn{4}{|c|}{ Position } & \multirow[b]{2}{*}{$\mathrm{L}$} & \multirow[b]{2}{*}{0} & \multicolumn{3}{|c|}{ Position } \\
\hline & & & 1 & 2 & 3 & 4 & 5 & & & 1 & 2 & 3 & 4 & & & 2 & 3 & 4 \\
\hline $\mathrm{Cl}$ & 3 & 3 & & 82.1 & 47.0 & 83.3 & & 3 & 3 & & 83.7 & 53.6 & 86.4 & 3 & 3 & 85.8 & 67.4 & 87.1 \\
\hline $\mathrm{C} 2$ & 3 & 5 & & & & & & 3 & 3 & & 91.4 & 52.9 & 87.4 & 3 & 3 & 91.3 & 63.2 & 94.1 \\
\hline E1 & 5 & 3 & & 97.6 & 93.2 & 96.3 & & 4 & 3 & & 95.0 & 82.4 & 94.4 & 3 & 3 & 92.8 & 75.3 & 87.6 \\
\hline $\mathrm{E} 2$ & 5 & 5 & 94.8 & 82.5 & 86.1 & 89.8 & 91.7 & 4 & 4 & 94.6 & 83.6 & 89.4 & 93.6 & 3 & 3 & 93.3 & 77.1 & 92.2 \\
\hline
\end{tabular}

Note-Data were not available for Group C2, Phase $I$ (see text). $L=$ the number of stimulus keys lit on each trial; $O=$ the number of different keys on which the odd stimulus could appear over trials. 
A repeated measures analysis of variance performed on the position data of Group $\mathrm{Cl}$ indicated a reliable effect of position $[F(2,6)=8.60]$. A planned comparison indicated that performance at the two ends of the display was significantly better than performance at the center of the display $[F(1,6)=17.19]$. A similar analysis performed on the position data of Group E1 indicated a nonsignificant effect of position $[F(2,6)=2.18]$, though differences may have been obscured by a performance ceiling. An analysis of the position data of Group E2 indicated a significant position effect $[F(4,12)=6.35]$, to which superior endposition performance contributed significantly [performance on the end keys vs. performance on the three center keys; $F(1,12)=16.68$ ]. Performance among the three middle keys did not differ significantly $[F(2,12)=3.68]$.

\section{Phase 2}

The Phase 2 data indicated that performance was better with four keys lit than with three keys lit, but there were only minor differences in performance between the two groups with three keys lit and between the two groups with four keys lit. The data from Phase 2 appear in Figure 2.

The drop in performance when birds were shifted from five keys lit (last session of Phase 1) to four keys lit (first session of Phase 2), which averaged $7.5 \%$, was not quite reliable $[\mathrm{t}(7)=2.28, \mathrm{p}=.06]$.

An analysis of variance performed on the data from the first session of Phase 2 indicated that the difference in performance between groups with four keys lit and three keys lit was not quite reliable $[F(1,12)=3.89, p=.07]$. The difference in performance between the two three-key-lit groups was also not reliable $(F<1)$, nor was the difference in performance between the two four-key-lit groups $[F(1,12)=2.23]$.

A similar analysis performed on the data pooled over the 12 Phase 2 sessions indicated that performance was better when four keys were lit than when three keys were lit $[F(1,12)=11.25]$, but the performance of the two three-key-lit groups did not differ significantly from each other, nor did the performance of the two four-key-lit groups (both Fs $<1$ ).

The position data from Phase 2 pooled over the last 10 sessions indicated $V$-shaped performances by Groups C1, C2, and E1. Performance by Group E2 was similarly higher at the ends than in the middle. The position data from the last 10 sessions of Phase 2 appear in Table 3.

A repeated measures analysis of variance performed on the position data indicated a significant effect of position for Group C1, Group E1, and Group E2 $[F(2,6)=6.77, F(2,6)=6.44, F(3,9)=3.91$, respectively].

\section{Phase 3}

The data from Phase 3 indicated that although there appeared to be an initial difference in performance between birds shifted to three keys lit and those that had always experienced three keys lit, the difference was not maintained over further sessions of experience with three keys lit. The data from Phase 3 appear in Figure 2.

An analysis of variance performed on the data from the first session of Phase 3 indicated that the difference in standard oddity performance between the groups that had been transferred from four keys lit and the control groups was not significant $[F(1,12)$ $=2.19$ ]. Similarly, the difference in performance between the two groups transferred from four keys lit was not significant, nor was the difference in performance between the control groups (both Fs $<1$ ).

A similar analysis performed on the data from the 10 Phase 3 sessions combined indicated that the transferred birds did not perform significantly better than the control birds $[\mathrm{F}(1,12)=1.09]$. Furthermore, performance by the two transferred groups did not differ significantly from each other, nor did performance by the two control groups (both Fs $<1$ ).

The position data from Phase 3 pooled over the 10 Phase 3 sessions indicated V-shaped functions for all four groups. The position data from the 10 sessions of Phase 3 appear in Table 3.

A repeated measures analysis of variance performed on the position data indicated a significant effect of position $[F(2,6)=10.94,4.17,9.55$, and 7.74 , for Groups E1, E2, C1, and C2, respectively]. The four groups showed comparable V-shaped performance functions.

\section{Discussion}

The results of Experiment 2, Phase 2 indicate that an increase in the number of incorrect alternatives facilitated oddity performance regardless of whether the odd stimulus appeared in all positions or not, over trials. Increasing the number of oddity positions from three to fjve had virtually no effect on the threekey oddity task, although it appeared to facilitate performance of the five-key oddity task. The latter effect may have been due to the fact that, for Group E1, although all five positions were lit, the odd stimulus never appeared on either end position; thus, the end positions consistently provided information concerning the incorrect color. The task for Group E1 thus became an oddity-from-sample task with either end key serving as the sample. Zentall et al. (1974) have shown that such an oddity-from-sample task (even without required orienting responses to the samples) is easier for pigeons to learn than is a standard oddity task.

As mentioned earlier, both the size-numberdiscrimination hypothesis and figure-ground hypothesis can account for better performance with five keys lit than with three keys lit. Increasing the number of 
incorrect alternatives increases the number (or area) of contiguous incorrect alternatives with which to compare the odd stimulus, and it probably also creates a "better" ground against which to perceive the figure (odd stimulus). The position data, however, suggest that the size-number hypothesis is correct. End-key performance by Group E2 was higher than performance on the three center keys. Furthermore, performance by Group E2 on the center three keys was comparable to end-key performance by Group $\mathrm{C} 1$, an outcome consistent with the size-numberdiscrimination hypothesis, but inconsistent with the figure-ground hypothesis.

The data from birds shifted from five keys lit to four keys lit (Phase 2) and from four keys lit to three keys lit (Phase 3 ) also offer some support for the sizenumber-discrimination hypothesis. Performance with four keys lit was superior to control performance, whereas birds transferred from four keys lit to three keys lit did not perform better than control birds with three keys lit. Center-odd trials are not be made any easier by prior exposure to four- and five-key displays. These data suggest that the problem with three-key-lit center-odd trials is a problem in perceptually integrating the common information on the two end keys.

On the other hand, the failure to observe a difference between experimental and control groups in Phase 3 may have been due to the high level of performance by control birds (over $80 \%$ correct). Differences between groups might have been obtained had control group performance been lower. The difficulty in interpreting nonsignificant group differences in Phase 3 notwithstanding, the bulk of the data from Experiment 2 suggest that pigeons learn a standard oddity task, involving up to five keys lit per trial, by making a size or number discrimination between the odd stimulus and the matching stimulus. Manipulations that make the size or number discrimination easier, such as increasing the number of incorrect alternatives, will thus tend to facilitate oddity acquisition.

\section{REFERENCES}

Gollin, E. S., Saravo, A., \& Salten, C. Perceptual distinctiveness and oddity-problem solving in children. Journal of Experimental Child Psychology, 1967, 5, 586-596.

Gollin, E. S., \& Schadler, M. Relational learning and transfer by young children. Journal of Experimental Child Psychology, 1972, 14, 219-232.

Korfk A, K. Principles of Gestalt psychology. New York: Harcourt, Brace \& World, 1935

Nissen, H. W., \& McCulloch, T. L. Equated and nonequated stimulus situations in discrimination learning by chimpanzees. III. Pre-potency of response to oddity through training. Journal of Comparative Psychology, 1937, 23, 377-381.

PAstore, N. Discrimination learning in the canary. Journal of Comparative and Physiological Psychology, 1954, 47, 389-390.

Zentall, T. R., Hogan, D. E., \& Holder, J. Comparison of two oddity tasks with pigeons. Learning and Motivation, 1974, 5, 106-117.

Zentall, T. R., \& Hogan, D. E. Same/different concept learning in the pigeon: The effect of negative instances and prior adaptation to transfer stimuli. Journal of the Experimental Analysis of Behavior, 1978, 30, 177-186.

(Received for publication December 19, 1979; revision accepted April 25, 1980.) 